\title{
Protection of Chickens with Maternal Avian Influenza Virus (AIV) Immunity after Vaccination with a Recombinant AIV-Newcastle Disease Vector ${ }^{\dagger}$
}

\author{
Magdalena Murr 1,*, Olayinka Asala ${ }^{2}$, Axel Karger ${ }^{1}$, Christian Grund ${ }^{2}$, Thomas C. Mettenleiter ${ }^{1}$ \\ and Angela Römer-Oberdörfer ${ }^{1}$ \\ 1 Institute of Molecular Virology and Cell Biology, Friedrich-Loeffler-Institute, Federal Research Institute for \\ Animal Health, Südufer 10, 17493 Greifswald-Insel Riems, Germany; Axel.Karger@fli.de (A.K.); \\ ThomasC.Mettenleiter@fli.de (T.C.M.); Angela.Roemer-Oberdoerfer@fli.de (A.R.-O.) \\ 2 Institute of Diagnostic Virology, Friedrich-Loeffler-Institute, Federal Research Institute for Animal Health, \\ Südufer 10, 17493 Greifswald-Insel Riems, Germany; ofcasala@gmail.com (O.A.); \\ Christian.grund@fli.de (C.G.) \\ * Correspondence: magdalena.murr@fli.de; Tel.: +49-38351-71629 \\ + Presented at Viruses 2020-Novel Concepts in Virology, Barcelona, Spain, 5-7 February 2020. \\ Published: 17 June 2020
}

\begin{abstract}
Highly pathogenic avian influenza virus (HPAIV) belongs to the Orthomyxoviridae family and causes a systemic and highly lethal disease in poultry. Vaccination with recombinant Newcastle disease vector viruses (NDV) expressing the hemagglutinin (HA) of HPAIV H5N1 induces high antibody titers in chickens free of specific pathogens, conveying protection against a lethal infection with HPAIV H5N1. Protection of chickens possessing maternally derived NDV immunity was achieved after the replacement of the surface proteins of NDV, the fusion protein $(\mathrm{F})$, and the hemagglutinin-neuraminidase protein $(\mathrm{HN})$ against those of avian paramyxovirus serotype 8 . However, maternal AIV antibodies ( $\alpha$ AIV-MDA+) still interfere with vaccine virus replication, resulting in inefficient protection. For our study, recombinant rNDVsolH5_H5 was generated. The insertion of a transgene encoding a truncated soluble HA between the NDV phosphoprotein and matrix protein genes - in addition to the gene encoding a membrane-bound HA inserted between the NDV, F and HN of the lentogenic NDV Clone 30 - was expected to increase the total amount of HA expressed by the recombinant virus. Western blot and mass spectrometry analyses confirmed the increase in HA expression compared to the parental rNDVH5 expressing only the full-length HA. The protective efficacy of the newly generated recombinant NDV was tested in an animal experiment. $\alpha$ AIV-MDA+ chickens were vaccinated either 7,14 , or 21 days after hatching. A homologous challenge infection was carried out three weeks later. Although the youngest chickens showed the highest titer of $\alpha$ AIV-MDA, there were no AIV antibodies detectable 21 days after vaccination. However, $40 \%$ of vaccinated chickens were protected, while $85 \%$ and $100 \%$ protection was observed in the middle-aged and oldest chickens, which had low and no detectable levels of $\alpha$ AIV-MDA, and moderate and high AIV antibody levels after vaccination, respectively. Challenge infection of non-vaccinated chickens resulted in high mortality.
\end{abstract}

Keywords: avian influenza, H5N1, Newcastle disease, vector vaccine, DIVA, maternal immunity

(C) 2020 by the authors. Licensee MDPI, Basel, Switzerland. This article is an open access article distributed under the terms and conditions of the Creative Commons Attribution (CC BY) license (http://creativecommons.org/licenses/by/4.0/). 